Sir,--I am very glad indeed that Mr. FitzGerald has taken up the discussion of the laws stated in my article in the January issue of the JournaL, for I quite agree with him that the more cases that can be included, under a certain law, the more useful does the law become.

The thorough " thrashing out" of "Law A," as applied to all devices for obtaining dynamic support from the atmosphere, and of "Law B" as applied to the inclined plane or aeroplane, is, at the present time, of great importance to the science of aerodynamics. If the laws are true and absolutely general, as I feel assured they are, they furnish a corner-stone on which to build both theory and practice. When we remember that there were locomotives 25 years before Stephenson's "Rocket" which was the prototype of all modern locomotives), we should not feel certain that we have, in the present-day aeroplane, the prototype of the dynamic flier of the future, that shall encompass aerial navigation, in the broadest sense, in any weather-the aeroplane we have, and it is a great step in advance; time alone, however, can decide whether it is the prototype of the future machine or not. In any case, it is of the greatest importance that the general laws of aerodynamics be formulated and understood as early as possible ; theory, laboratory experiments, and field practice should go "hand-in-hand " for rapid and thorough progress.

To return to Mr. FitzGerald's letter, I do not think that " Law B" can be properly called " the complement of Langley's law "- "Law B," as I understood the matter, is the complete general law (in regard to efficiency) of the inclined plane and aeroplane, and includes "Langley's law" (as heretofore known) as its first phase. This first phase might have an infinite ordinate ( $\mathrm{I}$ refer to Figs. 2, 4, and 6), if it were possible to conceive of a plane without thickness and without skin-friction; but if it has any thickness there is drift, and if it has any surface there is also drift due to skin-friction. We may imagine a plane infinitely thin, but we cannot imagine a plane without surface, as it would cease, by definition, to be a plane; it seems apparent, therefore, that even as a wholly theoretical case, "Langley's law" can only obtain as the first phase of "Law B," and this first phase can attain a maximum finite ordinate only.

Mr. FitzGerald draws attention to Lanchester's statement that "Langley's law" only holds true in the case of a plane infinitely thin, but it cannot hold true even for a plane infinitely thin, for we cannot have a plane without surface, as pointed out above. I think we are, therefore, justified in believing that "Law B," in its general form of expression, applies as well to the mathematical plane of no thickness, to the material plane with no supporting members (i.e., members that can be corrected for), and to the complete aeroplane with supporting members and with body and strut resistances of various kinds.

Mr. FitzGerald's deductions as to the mathematics of the curves are interesting, but I think we should be a little more exact in our statement concerning the formulæ. The curves in Figs. 2, 4, and 6 are obviously not common hyperrbolæ themselves, but the curve joining the maximum ordinates is apparently of hyperbolic form,* as $\mathbf{M r}$. FitzGerald shows. I do not think we can find suitable equations for the curves themselves (in Figs. 2, 4, and 6) applicable to the various cases ; both the first and second phases (in certain cases) remind one of the Cissoid, or possibly the Cycloid, but neither the equations of the Cissoid or Cycloid express even the first phase.

As regards the maximum lift curves of Figs. 3, 5, and 7, the equation of the parabola certainly fulfils the conditions with reasonable accuracy, when we take the curves between the limit of 200 and 1,000 sq. ft. per 1,000 lb.; but when we extend the limits to $100 \mathrm{sq}$. ft. the constant, M, will vary considerably from the average value.

Regarding the "speed at which maximum occurs" curves, of Figs. 3, 5, and 7, described by Prof. FitzGerald as " a kind of hyperbola." While the equation $\mathrm{SV}^{2}=\mathbf{N}$ seems to answer very well for the curves, between the limits of 200 and 1,000 sq. ft. per $1,000 \mathrm{lb}$., it does not apply so well when we extend the limits to $100 \mathrm{sq}$. $\mathrm{ft}$., as here again the constant, $N$, is subject to a considerable variation from the average value.

Before publishing the curves in the January issue of the JournaL, I examined them to obtain formulæ that would properly express them, and came to the conclusion that in our present state of knowledge, at least, it is perhaps better to abide by the curves rather than formulæ. To be sure, at the present time, the limits of nearly all practical aeroplanes lie between the limits of 200 to $1,000 \mathrm{sq}$. ft. per $1,000 \mathrm{lb}$., but it is difficult to say what the future may bring forth, and, if the curves more faithfully represent the

* The equation $x y=a$ constant, is that of a hyperbola referred to its asymptotes as axes. 
actual relationships than formulæ, they are to be preferred. Nevertheless, it is certainly important to keep the proposed formulæ in mind, more accurate experimental data may bring the curves and formulæ in closer accord and, if we find that formulæ can be properly applied to our aerodynamic curves, they furnish a very valuable means of generalising our ideas, and would help on the progress of practice as well as theory.

With respect to Langley's reason for adopting curve $B$, in preference to curve $A$ (Fig. 1 of the original article), Langley was undoubtedly justified in lowering curve A (between the angles of zero and twenty degrees) on account of the square-edged planes used,* but he was not justified in producing the curve to zero, for by so doing he ignored the effect of skin-friction, as previously pointed out. This may seem a very small matter to argue about, but it is precisely this small drift at low angles of inclination (which must always exist with any surface) that turns "Langley's law" into the first phase of the general " Law B." In actual flight the low angles of incidence occur at the highest speeds, and since the drift, as well as the lift, is affected by the $\mathrm{V}^{2}$ law, it makes a very material difference whether we accord to drift (at zero degrees) a positive or zero value.

Turning to Mr. FitzGerald's deductions for practical cases, I cannot quite agree with the idea that the speed of minimum horse-power is necessarily either inconvenient or dangerous (except, of course, as the question of speed applies to stability-a question always of importance). I would, however, be the last to advocate no reserve of power, as aeroplanes and motors stand to-day, but it is quite conceivable that an aeroplane be designed for a maximum load per H.P., and that a motor be so designed that it works at best efficiency (at the designed aeroplane efficiency and speed) while holding in reserve considerable additional power applicable, when desired, at the will of the operator, by throttle or other control. Moreover, as Mr. FitzGerald suggests, a normal speed between that of minimum horse-power and minimum resistance, may be found to work out best in practice.

In my article I purposely refrained from dealing with the question of speed of minimum resistance, as I hope to treat this subject in a subsequent paper, and my one mission was to place "Langley's law" in a clearer light, and to substitute the more complete law, which includes " Langley's law" as its first phase. It goes without saying, that the speed of minimum resistance, in contrast with that of minimum power, is a matter of much interest and importance, but there are certain cases where the two speeds lie very close together, and the whole question is on quite worthy of a special discussion.

I am glad that Mr. FitzGerald draws attention to the linear relation between minimum horse-power for a given weight and the corresponding speed, as the matter escaped my attention, but here again we must not jump to a too hasty conclusion. Upon plotting the results we find that the relation is well expressed, in the case of the plane surfaces, by a straight line ; but it is more accurately expressed, in the case of curved surfaces, by a curve; this is a matter that I propose discussing more fully in the article on the speeds of minimum resistance and horse-power.

I would like to take this opportunity of making some additions to the text of the original article that should make some portions a little clearer.

\section{Errata and Addenda.}

P. 45-1l. 19 and 28-after " infinitely thin," add : " and without skin-friction."

P. 46-l. 10-instead of " or miles per hour," read : " and miles per hour."

P. 46-1. 19-after " in the curves of Fig. 2," add : "It is to be particularly noted that in Figs. 3, 5, and 7, ordinates are pounds lift per H.P. for the maximum lift curve, and are also miles per hour for the speed curve."

P. 46-1. 6 from bottom-after " sustained," add : " this confirmation is shown graphically in Figs. 3, 5, and 7 by the curve marked speed at which maximum occurs."

P. 46-add to footnote:- "But Eiffel, in the more accurately drawn curves given in his subsequent work, La Resistance de l'Air et l'Aviation, Paris, 1910, does not produce his drift curve to zero. This would somewhat alter the shape of curves in Figs. 4 and 5, and make them more similar to the refigured Langley results of Figs. 2 and 3."

P. 48-line 10-after "Langley's law," add: "but is true of the Langley-phase as above defined."

Rothesay, N.B., Canada.

W. R. TURNBULL.

March 20th, 1912.

* Eiffel's plane and curved plate also had square edges, the thickness being $3 \mathrm{~mm}$. with plan dimensions of $900 \mathrm{~mm}$. by $150 \mathrm{~mm}$. 